\title{
Numerical modeling of tunable optofluidics lens based on combined effect of hydrodynamics and electroosmosis
}

\author{
Haiwang $\mathrm{Li}^{a}$, Teck Neng Wong ${ }^{a, *}$, Nam-Trung Nguyen ${ }^{a}$, John C. Chai ${ }^{b}$ \\ ${ }^{a}$ School of Mechanical and Aerospace Engineering, Nanyang Technological University, 50 \\ Nanyang Avenue, Singapore 639798, Singapore \\ ${ }^{b}$ Department of Mechanical Engineering, The Petroleum Institute, P.O. Box 2533, Abu Dhabi, \\ United Arab Emirates \\ *Corresponding author. Email address: mtnwong@ntu.edi.sg (T.N.Wong)
}

\begin{abstract}
A numerical model of liquid-core liquid-cladding optofluidics lens under the combined effect of hydrodynamics and electroosmosis are presented in this paper. In the numerical simulation, a combined formulation using only one set of conservation equations to treat both fluids are employed. The coupled electric potential equation and Navier-Stokes equation are solved using the finite volume method. The level-set method is used to capture the interface between the fluids. To overcome a weakness in the level-set method, the localized mass correction scheme is applied to ensure mass conservation. The validity of the numerical scheme is evaluated by comparing with the experimental results; numerical results highlight the electroosmotic effect; the combined effect of pressure driven and electroosmosis can form optically smooth interfaces with arc-shape between the cladding fluids and the core fluid. Under fixed cladding flow rates, the same electric field forms symmetric biconvex lens only. Different electric fields can form biconvex lens, plane-convex lens, and meniscus lens. The results also present the velocity profiles and flow fields of micro lens. There is a good agreement between numerical and experimental results.
\end{abstract}




\section{Introduction}

Fluorescence has been widely used in biochemistry and analytical chemistry to track or analyze the bio-molecules or chemicals without destructive. Traditional method is using the external mercury lamp with a filter set or a laser with a specific wavelength to excite the fluorescence; this system is complex and expensive. MEMS technique provides one method to integrate fluorescence excitation and detection into a chip to make the whole system costeffective and portable [1-4]. Microlenses, which can improve the performance of the fluorescence excitation and modified the light properties, are widely integrated into lab-on-a-chip (LOC) systems to improve the portability of optical components and make the system costeffective [5,6]. However, the focal length of the solid lens is not tunable and the smoothness of interface of the solid lens depends on the fabrication process.

Optofluidics, which combines the fields of microfluidics and micro-optics, is implemented by controlling liquid interfaces in microchannels. Due to the special advantages of fluids, liquid core, liquid cladding optofluidics lens has been developed [7-10]. In the micro-lens models [710], the smooth and perfect curve interface between immiscible fluids is used to form the microlens. The focus of a microlens can be modified by changing the flow rate ratio between cladding fluid and core fluid.

Most of previous works accomplish the tunable microlens using pressure driven flow [7-10]. When the focal point is required to approach the microlens, the flow rate ratio between cladding flow and core flow is so small that it cannot be achieved $[11,12]$. Another one, the relationship between flow rate ratio and focal point is very complex [13]. In order to move the focal point to the desired location, the flow rates should be modified frequently.

In micro systems, surface-to-volume ratio is large; electroosmosis will be more efficient than the ordinary pressure-driven flow. The electroosmosis effect has been used to control flow in micro systems [14-18]. Unfortunately, the energy of electroosmosis is small [16]. The flow is unstable when the electric field is too high [19]. In order to overcome the drawback of pure pressure driven and electroosmotic effect, the electroosmotic effect is used to control flow conditions under the fixed flowrate [18,20,21]. 
This paper numerically investigates a new design of a circular chamber to realize an optically smooth lens curvature; the curvature can be adjusted using the electroosmotic effect under fixed flowrates. The numerical results are verified by previous experiments [22]. Fig. 1(a) shows the schematic concept of this optofluidics lens. The two cladding streams (cladding fluid 1 and cladding fluid 3) are electrically conducting with high electroosmotic mobility, while the core stream (core fluid 2) is non-conducting with low electroosmotic mobility. At a given pressure gradient and electric fields applied along the conducting cladding fluids, electroosmotic forces control the curvature of the interfaces (interface 1-2 and interface 3-2) between the conducting cladding fluids and the core fluid. The curvatures of the interfaces depend on the directions and magnitudes of the applied electric fields.

This paper focuses on the fluidic aspect of the lens design under the combined effect of pressure driven and electroosmosis using the numerical method. Optical characteristics of this lens should be similar to that of a lens tunable by pressure-driven flow as reported in details by Song et al. [10]. In this paper, the level-set method is used to capture the interface between the fluids [23,24].

\section{Numerical simulation}

\subsection{Governing equations}

Fig. 1(a) shows the schematic of the problem considered in this article. The main part of the system is circular chamber with diameter of $1 \mathrm{~mm}$ and thickness of $0.05 \mathrm{~mm}$. The channel has the uniform thickness of $0.05 \mathrm{~mm}$. Fig. 1(b) shows the zone of simulation in numerical simulation. The circular chamber is filled with three immiscible fluids (two high electroosmotic mobility fluids and one low electroosmotic mobility fluid). The high electroosmotic mobility fluids 1 and 3 are introduced through the inlet 1 and inlet 3 respectively; the low electroosmotic mobility of fluid 2 is introduced through the inlet 2 . To illustrate the concept, the volumetric flow rate of the fluids are fixed, electric fields $\left(V_{x 1}\right.$ and $\left.V_{x 3}\right)$ are applied along the high

electroosmotic mobility fluids 1 and 3 , respectively. The flow is affected by the combined effect of pressure gradient and electroosmotic effect.

The steady state, incompressible continuity and momentum equations for three-Newtonian 
fluid flow under the combined effect of pressure gradient and electric field in Cartesian coordinate system can be written as

$$
\begin{aligned}
& \nabla \cdot\left(\rho_{i} v\right)=0 \\
& \nabla \cdot\left(\rho_{i} v v\right)-\mu_{i} \nabla^{2} v=-\nabla p+E \rho_{e}
\end{aligned}
$$

where $\nu$ is the velocity vector, $p$ is the pressure, $\rho$ and $\mu$ are the density and viscosity appropriate for the phase occupying the particular spatial location, $\rho_{\mathrm{e}}$ is the net electric charge density, $\mathbf{E}$ is the electric field intensity, and the subscript of $i$ means the fluids. The electric field, E, in Eq. (2) is related to the electric potential, $\varnothing$, through

$$
\mathbf{E}=-\nabla \phi
$$

The electric potential, $\emptyset$, can be decomposed into two parts for small Debye length and small zeta potential; these two parts are the potential due to the charge acquired at the wall $\psi$, and the potential due to the external electric field $\varnothing[25]$

$$
\phi=\psi+\varphi
$$

Furthermore, the potential due to the charge acquired at the wall $\psi$, is governed by Poisson equation as

$$
\nabla^{2} \psi=-\frac{\rho_{e}}{\varepsilon}
$$

and the potential due to the external electric field is governed by Laplace equation as

$$
\nabla^{2} \varphi=0
$$

where $\varepsilon$ is the electric permittivity of the fluids. For a symmetric electrolyte, the net electric charge density, $\rho_{\mathrm{e}}$, is given by the Boltzmann distribution based on the assumption of local 
thermodynamic equilibrium [26] as

$$
\rho_{e}=-2 z_{0} e n_{0} \sinh \left(\frac{z_{0} e \psi}{k_{b} T}\right)
$$

where $z_{0}$ is the valence of the ions, $e$ is elementary charge, $n_{0}$ is the reference value of the ion concentration, $k_{\mathrm{b}}$ is Boltzmann constant, and $T$ is the absolute temperature. The length scale and velocity scale of the flow are taken as $L_{r e f}$ and $U_{r e f}$, respectively. The potential $\emptyset$ is scaled by the value of the potential applied at the inlet $V_{i n}$. The independent variable $\mathbf{r}$ and dependent variables $\mathbf{v}, p, \psi$ and $\rho_{q}$ are expressed in terms of the corresponding dimensionless quantities (shown with an over bar) by

$$
\left\{\begin{array}{l}
\mathbf{r}=L_{r e f} \overline{\mathbf{r}} \\
\mathbf{v}=U_{r e f} \overline{\mathbf{v}} \\
\psi=k_{b} T \bar{\psi} /\left(z_{0} e\right) \\
p=\rho_{1} U_{r e f}^{2} \bar{p} \\
\rho_{e}=2 n_{0} z_{0} e \bar{\rho}_{e}
\end{array}\right.
$$

Substituting Eq. (7) into Eq. (8), we can obtain the Poisson-Boltzmann equation (Eq. (9)). For a small potential of $0<\bar{\psi}<1$, the right-hand side of Eq. (7) can be mathematically approximated as $\sinh (\overline{\bar{\psi}}) \approx \bar{\psi}$ [27]. The Possion-Boltzmann equation (Eq. (9)) can be approximated as $\nabla^{2} \bar{\psi}$ $=K^{2} \bar{\psi}$. According the definition of Eq. (8), the dimensionless form of the governing equations can be described as

$$
\left\{\begin{array}{l}
\nabla \cdot\left(\bar{\rho}_{i} \bar{v}\right)=0 \\
\nabla \cdot\left(\bar{\rho}_{i} \overline{\mathbf{v}} \overline{\mathbf{v}}\right)=-\nabla \bar{p}+\mathbf{G} \cdot \bar{\rho}_{e}+\frac{1}{\operatorname{Re}} \bar{\mu}_{i} \nabla^{2} \overline{\mathbf{v}} \\
\nabla^{2} \bar{\varphi}=0 \\
\nabla^{2} \bar{\psi}=K^{2} \bar{\psi} \\
\bar{\rho}_{e}=-\bar{\psi}
\end{array}\right.
$$

where $G$ is the non-dimensional effect of electric field defined as $G=\frac{2 z_{0} e n_{0} L_{r e f} E}{\rho_{r e f} U_{r e f}^{2}}$ 
$\operatorname{Re}$ is the Reynolds number defined as $\operatorname{Re}=\frac{\rho_{\text {ref }} U_{r e f} L_{r e f}}{\mu_{r e f}}, \mathrm{~K}$ is the electrical double layer parameter defined as $\mathrm{K}=\frac{L_{r e f}}{K_{D}}$, where $\frac{1}{K}=\left(\frac{\varepsilon k_{b} T}{2 Z_{0}^{2} e^{2} n_{0}}\right)^{\frac{1}{2}}$.

\subsection{Boundary conditions}

The boundary conditions of velocities and electric potential are presented in Fig. 2. They can be described as follows

(at the inlet 1) $\left\{\begin{array}{l}u=0 \\ \phi=V_{1, \text { in }} \\ Q_{1, \text { in }}=v_{1, \text { in }} \cdot w\end{array}\right.$

(at the inlet 2) $\left\{\begin{array}{l}Q_{2, i n}=u_{2, i n} \cdot h \\ v=0 \\ \phi=0\end{array}\right.$

(at the inlet 3) $\left\{\begin{array}{l}u=0 \\ Q_{3, \text { in }}=v_{3, \text { in }} \cdot w \\ \phi=V_{3, \text { in }}\end{array}\right.$

(at the wall) $\left\{\begin{array}{c}u=0 \\ v=0 \\ \frac{\partial \phi}{\partial n}=0 \\ \psi=\xi_{i}\end{array}\right.$

where $\xi_{\mathrm{i}}$ is the zeta potential of the wall

(at the outlet 1) $\left\{\begin{array}{l}Q_{1, \text { out }}=Q_{1, \text { in }} \\ v=0 \\ \phi=V_{1, \text { out }}\end{array}\right.$ 
(at the outlet 2) $\left\{\begin{array}{l}Q_{2, \text { out }}=Q_{2, \text { in }} \\ v=0 \\ \phi=0\end{array}\right.$

(at the outlet 3) $\left\{\begin{array}{l}Q_{3, \text { out }}=Q_{3, \text { in }} \\ v=0 \\ \phi=V_{3, \text { out }}\end{array}\right.$

where $u$ is the velocity in $x$-axis and $v$ is the velocity in $y$-axis, $u_{2, i n}$ is the average velocity of inlet $2, v_{1, \text { in }}$ and $v_{3, \text { in }}$ are the average velocities in inlet 1 and inlet 3 respectively, $V_{1, \text { in }}$ and $V_{3, \text { in }}$ are the electric voltages in inlet 1 and inlet 3 respectively, $Q_{1, \text { in }}, Q_{2, \text { in }}$ and $Q_{3, \text { in }}$ are the volume flow rates of inlets respectively, $Q_{1, i n}, Q_{2, \text { in }}$ and $Q_{3, \text { in }}$ are the volume flow rates of outs respectively, $w$ is the width of inlet 1 and inlet $3, h$ is the height of inlet 2 (Fig. 1(b)).

\subsection{Interface definition}

The level-set method is applied to treat the interfaces between different fluids [23]. The level-set function is defined as

$\vartheta= \begin{cases}-d & \mathbf{r} \in \Omega_{\text {high electrical mobility fluids }} \\ 0 & \mathbf{r} \in \Gamma_{\text {interface }} \\ d & \mathbf{r} \in \Omega_{\text {low electrical mobility fluids }}\end{cases}$

where $\mathbf{r}$ is the position vector in the computational domain $\Omega, \Gamma$ is the interface, and $d$ is the shortest normal distance from the interface $\Gamma$.The governing equation for the evolution of the level-set function is described as

$$
\mathbf{v} \cdot \nabla \vartheta=0
$$

In the solution of Eq. (18), any convenient reference value can be assigned to the interface. The value of $\vartheta$ at all node points can be calculated based on the reference value at the interface. The level-set function $\vartheta$ is the normal distance from the interface and satisfies

$$
\nabla \vartheta=1
$$


Eq. (17) shows that the value of $\vartheta$ is set to zero at an interface and $\vartheta$ has opposite signs in adjacent liquids. In order to work properly, must remain the function of Eq. (19). However, the function of Eq. (19) can only be ensured at the beginning of the iteration process where the location of the interface is assumed and the values of $\vartheta$ at all nodes are specified. During the iteration process, the values of $\vartheta$ are calculated using Eq. (18). Although the interfaces are still represented by the reference values, the other values of $\vartheta$ may not be the shortest distance to the interface. In order to maintain the properties of level-set function, another scalar variable is defined and solved. This variable must be a distance function and has the same interface value as $\vartheta$. This scalar variable is defined as

$$
\frac{\partial \varsigma}{\partial \tilde{t}}=\operatorname{sign}(\vartheta)(1-|\nabla \vartheta|)
$$

where $\tilde{t}$ is a pseudo time for the variable $\varsigma$. The value of sign is given by

$$
\operatorname{sign}(\vartheta)=\frac{\vartheta}{\sqrt{\vartheta^{2}+|\nabla \vartheta|^{2}(\Delta x)^{2}}}
$$

where $\Delta x$ is the grid size. The steady state solution of $\varsigma$ always satisfies the properties of levelset function. The initial condition of Eq. (20) is

$$
\varsigma(r, 0)=\vartheta(r)
$$

Although the variable $\varsigma$ satisfies the level-set function, it suffers a significant drawback that the conservation of mass for the various phase cannot be ensured. To ensure the mass conservation at each cross section, a local mass correction factor is defined and an additional equation is solved as [28]

$$
\frac{\partial \varsigma^{\prime}}{\partial \tilde{\tilde{t}}}=\dot{m}_{c o r}
$$

In Eq. (23), $\tilde{\tilde{t}}$ is pseudo-time and can be different from the pseudo-time of $\tilde{t}, \dot{m}$ cor is the mass 
conservation factor. The mass conservation factor is calculated using the following equation

$$
\dot{m}_{\text {cor }}=\operatorname{sign}(\vartheta) \frac{\left(\dot{m}_{d}-\dot{m}_{c}\right)}{\dot{m}_{d}}
$$

where $\dot{m}_{\mathrm{d}}$ is the desired mass flow rate and $\dot{m}_{\mathrm{c}}$ is the most current local mass flow rate of the reference phase. The mass flow rate of the reference phase can be calculated using the following equation

$$
\dot{m}= \begin{cases}\sum \rho_{\text {ref }}\left(1-H_{\gamma}\right) u \Delta A & H_{r, \text { ref }}=0 \\ \sum \rho_{\text {ref }} H_{\gamma} u \Delta A & H_{r, \text { ref }}=1\end{cases}
$$

where $\Delta A$ is the cross-section of one grid and the summation is performed over a cross-section. In this paper, the desired mass flow rate is calculated using the inlet conditions.

\subsection{Properties}

The density $\rho$, the dynamic viscosity $\mu$ and the electric permittivity $\varepsilon$ are calculated using

$$
\alpha=\left(1-H_{r}\right) \alpha_{\text {low electrical mobility fluid }}+H_{r} \alpha_{\text {high electrical mobility fluid }}
$$

where $\alpha$ means the density $\rho$, the dynamic viscosity $\mu$ and the electric permittivity $\varepsilon$.The Heaviside function $\mathrm{Hr}$ is related to the normal distance from the interface and is calculated [23]

$$
H_{\gamma}= \begin{cases}0 & \text { for } \vartheta<-\gamma \\ \frac{\vartheta+\gamma}{2 \gamma}+\frac{1}{2 \pi} \sin \left(\frac{\pi \vartheta}{\gamma}\right) & \text { for }|\vartheta| \leqslant \gamma \\ 1 & \text { for } \vartheta>\gamma\end{cases}
$$

where $\gamma=1.5 \Delta \mathrm{x}$ [23].

\subsection{Depth average method}

The size of model along $x$ direction and $y$ direction is larger than that of $z$ direction. The velocity profile in $z$ direction is shown in Fig. 3: the electroosmotic velocity of cross section 
along $z$ direction is plug profile [18,25,29], it is function of electric field $\bar{u}^{E}=\int u^{E} d z=f(E)$; the pressure driven velocity is parabolic profile, it is function of pressure gradient $\bar{u}^{p}=\int u^{p} d z=f(p)$. The depth average method [30] is used in this paper to reduce the $3 \mathrm{D}$ model to $2 \mathrm{D}$ model.

\subsection{Solution procedure}

The solution procedure can be summarized as follows

(1) Guess the locations of the interfaces.

(2) Calculate the value of $\vartheta$ for all nodes for the interface.

(3) Calculate the properties for all nodes using Eqs. (26) and (27).

(4) Solve the distribution of electric potentials using Eq. (9).

(5) Calculate the average velocity using the depth average method.

(6) Solve the continuity and momentum equations given by Eq. (9).

(7) Calculate the value of $\vartheta$ using Eq. (18) according the velocity distribution of step (6).

(8) Calculate the value of $\varsigma$ according the solution of step (7).

(9) Calculate the value of $\varsigma^{\prime}$ 'using Eq. (23) according the solution of step (8).

(10) Set $\vartheta(r)=\varsigma^{\prime}(r)$.

(11) Repeat steps (3)-(10) until the solution converges.

\subsection{Numerical method}

The continuity equation, momentum equation, electric potential equations, and level-set equations can be described using the following equations

$\rho u_{j} \frac{\partial \Phi}{\partial x_{j}}=\frac{\partial}{\partial x_{j}}\left(\Gamma \frac{\partial \Phi}{\partial x_{j}}\right)+S$ 
where $\Phi, \rho, \Gamma$ and $S$ are the dependent variables, density, diffusion coefficient and source term respectively. Eq. (28) is solved using the finite-volume method of Patankar [31]. A staggered grid is used in this article. The scalar variables are stored at the centers of the control volumes, and the velocities are located at the control volume faces. In this paper, the power-law is used to calculate the combined convection-diffusion effect in the momentum equations. The convection of the level-set equations is modeled by the first-order unwind scheme. The velocitypressure coupling is resolved using SIMPLER algorithm. The transient term is discredited using the fully implicit scheme. The resulting algebraic equations are solved using the TriDiagonal Matrix Algorithm.

\section{Validation of the numerical scheme}

\subsection{The effect of grid resolution}

The interface shape and positions in the micro chamber using various computational grids are shown in Fig. 4. Uniform grid is used in the simulations. In Fig. 4, the value of $x$-axis means the grid number in circular chamber.

In the simulations, the width of the inlets $(w) 1$ and 3 are $100 \mu \mathrm{m}$; the height of the inlet 2 is $100 \mu \mathrm{m}$; the diameter of the circular chamber (D) is $1 \mathrm{~mm}$. The voltages added to cladding fluids are $V_{x 1}=V_{x 3}=800 \mathrm{~V}$. The flowrates of cladding fluids and core fluid are $q_{1}=q_{2}=q_{3}=0.05$ $\mathrm{ml} / \mathrm{h}$. The properties of the fluid 1 are reference quantities. The permittivity of conduction fluid is $6.9 \times 10^{-1}\left(\mathrm{C}^{2} \mathrm{~N}^{-1} \mathrm{~m}^{-2}\right)$ [29]. All fluids have the same viscosity $\mu_{1}=\mu_{2}=\mu_{3}=0.85 \times 10^{-3}$ $\mathrm{Ns} / \mathrm{m}^{2}$. The reference length is $L_{r e f}=D$; the reference velocity is the average velocity of fluid 1 of $U_{r e f}=-Q_{1, i n} / w$ and the Reynolds number is about $3 \times 10^{-4}$.

The results presented in Fig. 4 show the decay of discretization error with increased the number of grid. It can be seen that the exact solution [22] is reproduced accurately when the grid number in circular chamber exceeds $17,000^{2}$. It means that the interface evolution between immiscible fluids is well captured and the numerical method is suitable. In the following simulation, the grid number in circular chamber is $17,000^{2}$.

\subsection{Validation of numerical results}


The numerical model presented in the previous section is validated by comparing with the experimental results [22]. The parameters are the same as Section 3.1.

Fig. 5 compares the measured interface shapes with that of simulation results. The agreement between the experimental and numerical results confirms that the numerical model is suitable to predict the interface positions under different voltage. The results also comparing with analytical model of our previous work [22]; the agreements between experimental results, numerical results and analytical model confirms that numerical scheme of this paper can predict the flow conditions and interface shape using electroosmotic effect under fixed flowrates.

\section{Results and discussion}

\subsection{Formation of biconvex lens, plan-convex lens, and meniscus lens}

We define the field of the core fluid 2 as a region, the interface 1-2 and interface 3-2 are the boundaries of the region, and the curvature of the interface is defined as the divergence of the normal of the interface. The symbol of the curvatures can be defined as $\kappa>0$ for the convex region and $\kappa<0$ for the concave region (Fig. 6).

The curvature of interface $1-2 \kappa_{\left({ }_{1-2}\right)}$ and the curvature of the interface $3-2{ }_{\left(\kappa_{3-2)}\right.}$ can be calculated using the following formula (Fig. 7):

$$
\left\{\begin{array}{l}
\kappa_{1-2}=-\frac{2 B_{2}}{B_{2}^{2}+R^{2}} \\
\kappa_{3-2}=\frac{2 A_{2}}{A_{2}^{2}+R^{2}}
\end{array}\right.
$$

where $R$ is the radius of the interface.

Fig. 7 shows a series of different optofluidics lenses simulated by the proposed numerical model. The electroosmotic effect for controlling optofluidics lens is confirmed numerically. Figs. 7(a) - (f) shows that at a fixed flow rate, the curvatures of the interfaces (1-2 and 3-2) can be modified according to the magnitudes and the polarities of the voltages.

If positive voltages are added to the cladding fluids synchronously, (Fig. 7(b)), the 
electroosmotic flow acts in the same direction with the flow direction. Consequently, $q_{1}$ encounters a lower resistance. $q_{1}$ appear to be less "viscous" due to the electroosmotic effect. Because of the same pressure drop along the microchannel and the fixed volumetric flow rate, the lower viscous fluid has to focus into a smaller area, thus decreases the curvature of the interface. Opposite with positive voltage, the negative electric field appears to be more "viscous" (Fig. 7(a)). Figs. 7(a) and (b) show that a symmetric biconvex lens can be achieved when symmetric voltages $\left(V_{x 1}=V_{x 3}\right)$ are added to the cladding fluids.

When the applied voltages are different $\left(V_{x 1} \neq V_{x 3}\right)$, the curvatures of the interface 1-2 and the interface 2-3 have different values (Fig. 7(c)-(f)). Fig. 6 defines the symbol of curvature and the type of the lens.

The above results show that a negative voltage could decrease the curvature of the interface, while a positive voltage can increase the curvature of the interface. Under fixed flow rates, the same voltage achieves symmetric biconvex lens only. Different voltages can form biconvex lens, plano-convex lens, and meniscus lens. The control voltage can be further reduced working with a lower flow rate [29].

\subsection{Biconvex lens, plan-convex lens, and meniscus lens: Comparison between numerical simulation and experiment}

Fig. 8 compares the measured curvature of the interface and the numerical results when the voltages have opposite polarity $\left(V_{x 1}=-V_{x 3}\right) . \mathrm{A}_{2}$ and $\mathrm{B}_{2}$ of Fig. 8 are defined in Fig. 9. The results clearly show that the numerical model agrees well with the measurement. Fig. 8 shows that under constant flow rates, different optofluidics lenses can be achieved but a combination of the positive and negative voltages is needed. If there is no voltage applied, the flow is purely pressure driven. If the cladding flows have the same rate, a symmetric biconvex lens is achieved. When different voltages are added to the cladding flows, $\kappa_{2}$ and $\kappa_{3-2}$ have different values. If the magnitude of the voltage is not high enough (e.g. $-200 \mathrm{~V}<V_{x l}<200 \mathrm{~V}$ ), $\kappa_{1-2}$ and $\kappa_{3-2}$ are positive at the same time forming a biconvex. $\kappa_{1-2}$ or $\kappa_{3-2}$ decreases with the increase of voltages. A plano-convex lens is formed when $\kappa_{1-2}=0$ or $\kappa_{3-2}=0$ (e.g. $V_{x 1}=-200 \mathrm{~V}$ or $V_{x 3}=-$ $200 \mathrm{~V}$ ). When $\kappa_{1-2}$ or $\kappa_{3-2}$ is negative with an increasing magnitude of voltages (e.g. $\left|V_{x 1}\right|>$ $200 \mathrm{~V}$ ), a meniscus lens is formed. The above results show that under a constant flow rate, a 
biconvex lens, a plano-convex lens, and a meniscus lens can be accomplished.

\subsection{Velocity fields of center line for different lens model}

The detail of velocity fields in circular chamber of biconvex lens and a meniscus lens are shown in Fig. 10(a) and Fig. 11(a) respectively. The velocities at the inlet and outlet of the circular chamber are highest due to its smallest cross section. While Figs. 10(b) and 11(b) show the corresponding velocity $u$ at the center of the chamber. $u^{p}$ is the velocity component corresponding to hydrodynamic velocity; $u^{E}$ is the velocity component corresponding to electroosmotic velocity.

Fig. 10(b) shows that the velocity distribution is symmetric due to the symmetrical of the biconvex lens. In the analysis, $\mu_{1}=\mu_{2}=\mu_{3}$. The flow characteristics depend on the coupling effects of the cladding fluids and core fluid 2 which involve the electrokinetics driving force in the cladding fluids and the interfacial phenomenon. The component of velocity is zero at the center line of the chamber. $u^{p}$ is plug profile because the viscosity of fluids has the same values and the aspect ratio is very large [32]. For $u^{E}$, when electric fields are applied across the cladding fluids, the cladding fluids 1 and 3 are driven by electroosmosis, which drags the core fluid 2 by the hydrodynamic shear force. The velocity field is affected by viscosity ratios, the strength of the external electric fields and electroosmotic characteristics of the cladding fluids. The electroosmotic velocity profile $\left(u^{E}\right)$ of the core fluid 2 is negative. It is due to the interfacial shear stress dragged by the cladding fluids on the core fluid 2. $u$ is the superposition of the solutions of $u^{p}$ and $u^{E}$.

Fig. 11(b) shows the velocity profile of the meniscus lens. $u^{E}$ changes dramatically due to the different applied electric field. Under the negative electric fields, the electroosmotic velocity is negative. Hence, the velocity profile is asymmetrical. The result indicates that flow fields can be modified by electric fields under same flow rates.

\section{Conclusion}

This paper presents the numerical method of a liquid-core liquid-cladding optofluidics lens 
tunable by the combined effect of hydrodynamics and electroosmosis. In this lens, an electrically conducting liquid is used as the cladding fluids, and the core fluid is nonconducting. Voltages are added to the cladding fluids. Under the constant flow rates, a combined formulation using only one set of conservation equations to treat both fluids are employed. The coupled electric potential equation and Navier-Stokes equation are solved together using finite volume method. The level-set method is used to capture the interface between the fluids. To overcome a weakness in the level-set method, the localized mass correction scheme is applied to ensure mass conservation. The results show that the velocity fields can be adjusted by electric fields under fixed flow rates. The interfaces between the cladding fluids and the core fluid are optically smooth arc-shape under the combined effect of hydrodynamic and electroosmosis. The curvature of interface can be controlled by external electric fields. Under the constant flow rates, the symmetric electric fields result in a symmetric biconvex lens only, while asymmetric electric fields form biconvex lens, plano-convex lens, and meniscus lens. The experimental results agree well with the numerical results.

\section{Acknowledgements}

T.N. Wong, N.T Nguyen and H. Li gratefully acknowledge research support from the Singapore Ministry of Education Academic Research Fund Tier 2 research Grant MOE2011T2-1-036. 


\section{References}

[1] M.L. Chabinyc, D.T. Chiu, J.C. McDonald, A.D. Stroock, J.F. Christian, A.M. Karger, G.M. Whitesides, An integrated fluorescence detection system in poly(dimethylsiloxane) for microfluidic applications, Anal. Chem. 73 (18) (2001) 4491-4498.

[2] S. Qi, X. Liu, S. Ford, J. Barrows, G. Thomas, K. Kelly, A. McCandless, K. Lian, J. Goettert, S.A. Soper, Microfluidic devices fabricated in poly(methyl methacrylate) using hot-embossing with integrated sampling capillary and fiber optics for fluorescence detection, Lab on a Chip - Miniaturisation for Chemistry and Biology 2 (2) (2002) 8895.

[3] A. Fonseca, I.M. Raimundo Jr, J.J.R. Rohwedder, R.S. Lima, M.C.U. Araújo, A microfluidic device with integrated fluorimetric detection for flow injection analysis, Anal. Bioanal. Chem. 396 (2) (2010) 715-723.

[4] S. Kühn, B.S. Phillips, E.J. Lunt, A.R. Hawkins, H. Schmidt, Ultralow power trapping and fluorescence detection of single particles on an optofluidic chip, Lab on a Chip Miniaturisation for Chemistry and Biology 10 (2) (2010) 189- 194.

[5] S. Camou, H. Fujita, T. Fujii, PDMS 2D optical lens integrated with microfluidic channels: principle and characterization, Lab on a Chip - Miniaturisation for Chemistry and Biology 3 (1) (2003) 40-45.

[6] J. Wenger, D. Gerard, H. Aouani, H. Rigneault, Disposable microscope objective lenses for fluorescence correlation spectroscopy using latex microspheres, Anal. Chem. 80 (17) (2008) 6800-6804.

[7] S.K.Y. Tang, C.A. Stan, G.M. Whitesides, Dynamically reconfigurable liquid-core liquid-cladding lens in a microfluidic channel, Lab on a Chip - Miniaturisation for Chemistry and Biology 8 (3) (2008) 395-401.

[8] M. Rosenauer, M.J. Vellekoop, 3D fluidic lens shaping - a multiconvex hydrodynamically adjustable optofluidic microlens, Lab Chip 9 (8) (2009) 1040-1042. 
[9] C. Song, N.T. Nguyen, A.K. Asundi, S.H. Tan, Tunable micro-optofluidic prism based on liquid-core liquid-cladding configuration, Opt. Lett. 35 (3) (2010) 327-329.

[10] C. Song, N.T. Nguyen, S.H. Tan, A.K. Asundi, A tuneable micro-optofluidic biconvex lens with mathematically predictable focal length, Microfluid. Nanofluid. (2010) 1-8.

[11] G.B. Lee, C.I. Hung, B.J. Ke, G.R. Huang, B.H. Hwei, Micromachined pre-focused 1 x $\mathrm{N}$ flow switches for continuous sample injection, J. Micromech. Microeng. 11 (5) (2001) $567-573$.

[12] G.B. Lee, B.H. Hwei, G.R. Huang, Micromachined pre-focused M x N flow switches for continuous multi-sample injection, J. Micromech. Microeng. 11 (6) (2001) 654-661.

[13] C. Song, N.T. Nguyen, S.H. Tan, A.K. Asundi, Modelling and optimization of micro optofluidic lenses, Lab on a Chip - Miniaturisation for Chemistry and Biology 9 (9) (2009) 1178-1184.

[14] A. Manz, C.S. Effenhauser, N. Burggraf, D.J. Harrison, K. Seiler, K. Fluri, Electroosmotic pumping and electrophoretic separations for miniaturized chemical analysis systems, J. Micromech. Microeng. 4 (4) (1994) 257-265.

[15] L. Ren, C. Escobedo, D. Li, Electroosmotic flow in a microcapillary with one solution displacing another solution, J. Colloid Interf. Sci. 242 (1) (2001) 264- 271.

[16] D. Sinton, D. Li, Electroosmotic velocity profiles in microchannels, Colloids Surf., A $222(1-3)(2003) 273-283$.

[17] D. Kohlheyer, G.A.J. Besselink, R.G.H. Lammertink, S. Schlautmann, S. Unnikrishnan, R.B.M. Schasfoort, Electro-osmotically controllable multi-flow microreactor, Microfluid. Nanofluid. 1 (3) (2005) 242-248.

[18] H. Li, T.N. Wong, N.T. Nguyen, Electroosmotic control of width and position of liquid streams in hydrodynamic focusing, Microfluid. Nanofluid. (2009) 1-9.

[19] Y.J. Pan, C.M. Ren, R.J. Yang, Electrokinetic flow focusing and valveless switching 
integrated with electrokinetic instability for mixing enhancement, J. Micromech. Microeng. 17 (4) (2007) 820-827.

[20] C. Wang, Y. Gao, N.T. Nguyen, T.N. Wong, C. Yang, K.T. Ooi, Interface control of pressure-driven two-fluid flow in microchannels using electroosmosis, J. Micromech. Microeng. 15 (12) (2005) 2289-2297.

[21] R.J. Yang, C.C. Chang, S.B. Huang, G.B. Lee, A new focusing model and switching approach for electrokinetic flow inside microchannels, J. Micromech. Microeng. 15 (11) (2005) 2141-2148.

[22] H. Li, T.N. Wong, N.T. Nguyen, A tunable optofluidic lens based on combined effect of hydrodynamics and electroosmosis, Microfluid. Nanofluid. 10 (5) (2011) 1033-1043.

[23] R.F. Stanley Osher, Level Set Method and Dynamic Implicity Surfaces, Springer Verlag New York, Inc, New York, 2003.

[24] A. Ganoun, R. Canals, A new fast level set method, in: Report - Helsinki University of Technology, Signal Processing Laboratory (2004) 232-235.

[25] N.A. Patankar, H.H. Hu, Numerical simulation of electroosmotic flow, Anal. Chem. 70 (9) (1998) 1870-1881.

[26] R.J. Hunter, Zeta potential in colloid science principles and applications, Harcourt Brace Jovanovich, Sydney, 1981.

[27] H.J. Keh, H.C. Tseng, Transient electrokinetic flow in fine capillaries, J. Colloid Interf. Sci. 242 (2) (2001) 450-459.

[28] Y.F. Yap, J.C. Chai, K.C. Toh, T.N. Wong, Y.C. Lam, Numerical modeling of unidirectional stratified flow with and without phase change, Int. J. Heat Mass Transfer 48 (3-4) (2005) 477-486.

[29] Y. Gao, C. Wang, T.N. Wong, C. Yang, N.T. Nguyen, K.T. Ooi, Electro-osmotic control of the interface position of two-liquid flow through a microchannel, J. Micromech. 
Microeng. 17 (2) (2007) 358-366.

[30] Y.C. Lam, X. Chen, C. Yang, Depthwise averaging approach to cross-stream mixing in a pressure-driven microchannel flow, Microfluid. Nanofluid. 1 (3) (2005) 218-226.

[31] S.V. Patankar, Numerical Heat Transfer and Fluid Flow, Hemisphere Pub. Corp., Washington, 1980.

[32] J.M. Chen, T.L. Horng, W.Y. Tan, Analysis and measurements of mixing in pressuredriven microchannel flow, Microfluid. Nanofluid. 2 (6) (2006) 455- 469. 


\section{List of Figures}

Figure 1 (a) Schematic representation of the optofluidics lens under the combined effect of hydrodynamic and electroosmosis; (b) the domain of mulation.

Figure 2 The boundary conditions of simulation.

Figure 3 The velocity profile in $z$ direction.

Figure 4 The effect of grid resolutions on errors between analytical solution [22] and numerical simulation.

Figure 5 The shape of the interface under the different electric fields.

Figure 6 The definition of the symbol of curvature and the model of lens. The core fluid is regarded as a region. Convex region has $\kappa>0$, concave region has $\kappa>0$, and a plan has $\kappa=0$. (a) Biconvex lens; (b) Meniscus lens; (c) Plano-convex lens; (d) Meniscus lens.

Figure 7 The fluid-fluid lenses based on the difference electric fields $\left(q_{1}=q_{2}=q_{3}=0.05 \mathrm{ml} / \mathrm{h}\right)$
(a) $V_{x l}=-100 \mathrm{~V} ; V_{x 3}=-100 \mathrm{~V}$;
(b) $V_{x l}=600 \mathrm{~V} ; V_{x 3}=600 \mathrm{~V}$;
(c) $V_{x l}=300 \mathrm{~V} ; V_{x 3}=-300 \mathrm{~V}$;
(d) $V_{x l}=-300 \mathrm{~V}$;
(e) $V_{x l}=-600 \mathrm{~V} ; V_{x 3}=600 \mathrm{~V}$;
(f) $V_{x l}=600 \mathrm{v} ; V_{x 3}=-600 \mathrm{~V}$.

Figure $8 \quad$ A2 and B2 with different voltages $\left(q_{1}=q_{2}=q_{3}=0.05 \mathrm{ml} / \mathrm{h}, V_{x 1}=-V_{x 3}\right)$.

Figure 9 The interface positions of a lens.

Figure 10 (a) Velocity field of the circular chamber; (b) velocity profile of center line of circular chamber $\left(q_{1}=q_{2}=q_{3}=0.05 \mathrm{ml} / \mathrm{h} ; V_{x 1}=-100 \mathrm{~V} ; V_{x 3}=-100 \mathrm{~V}\right)$.

Figure 11 (a) Velocity field of the circular chamber; (b) velocity profile of center line of circular chamber $\left(q_{1}=q_{2}=q_{3}=0.05 \mathrm{ml} / \mathrm{h} ; V_{x 1}=600 \mathrm{~V} ; V_{x 3}=-600 \mathrm{~V}\right)$. 

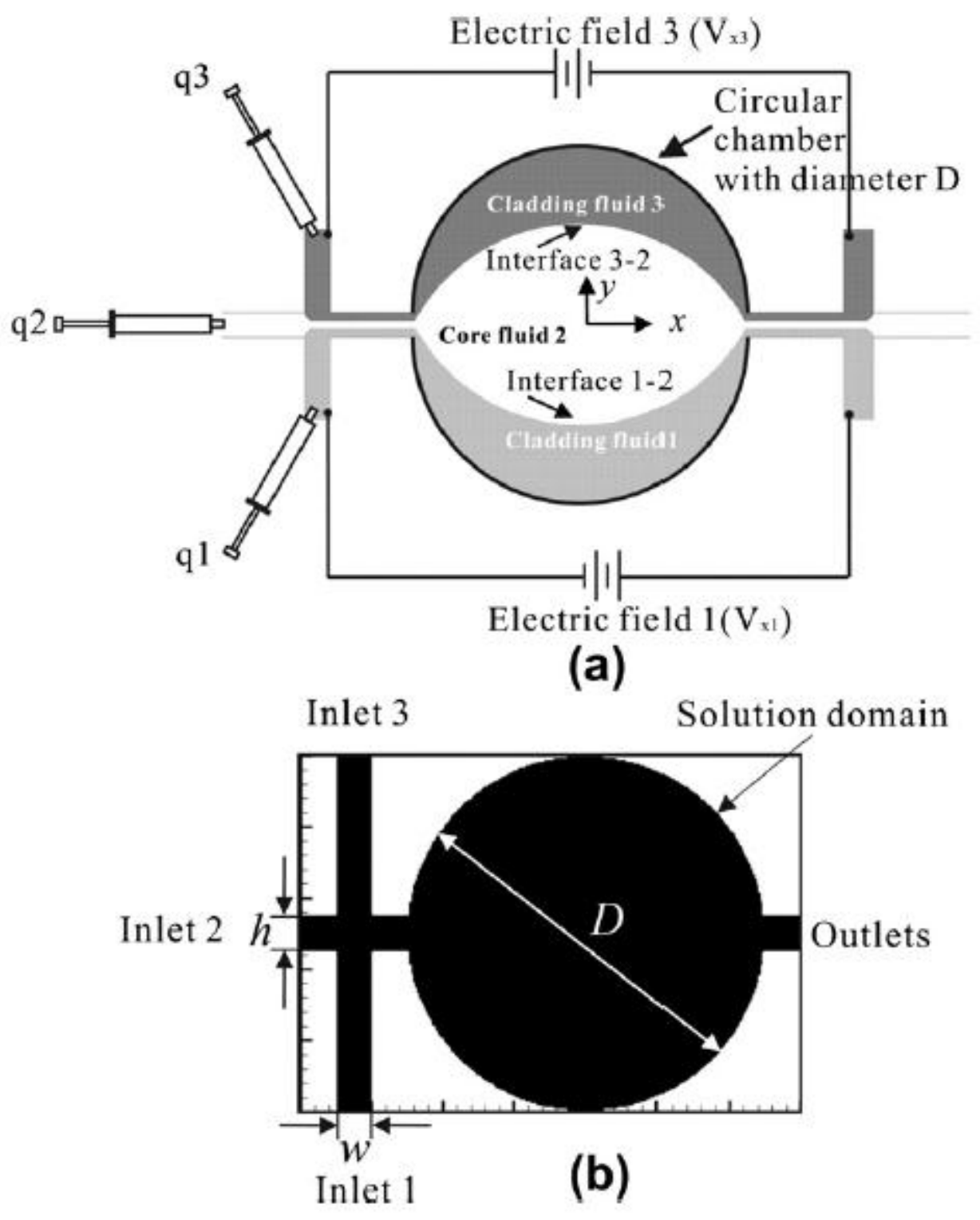

Figure 1 


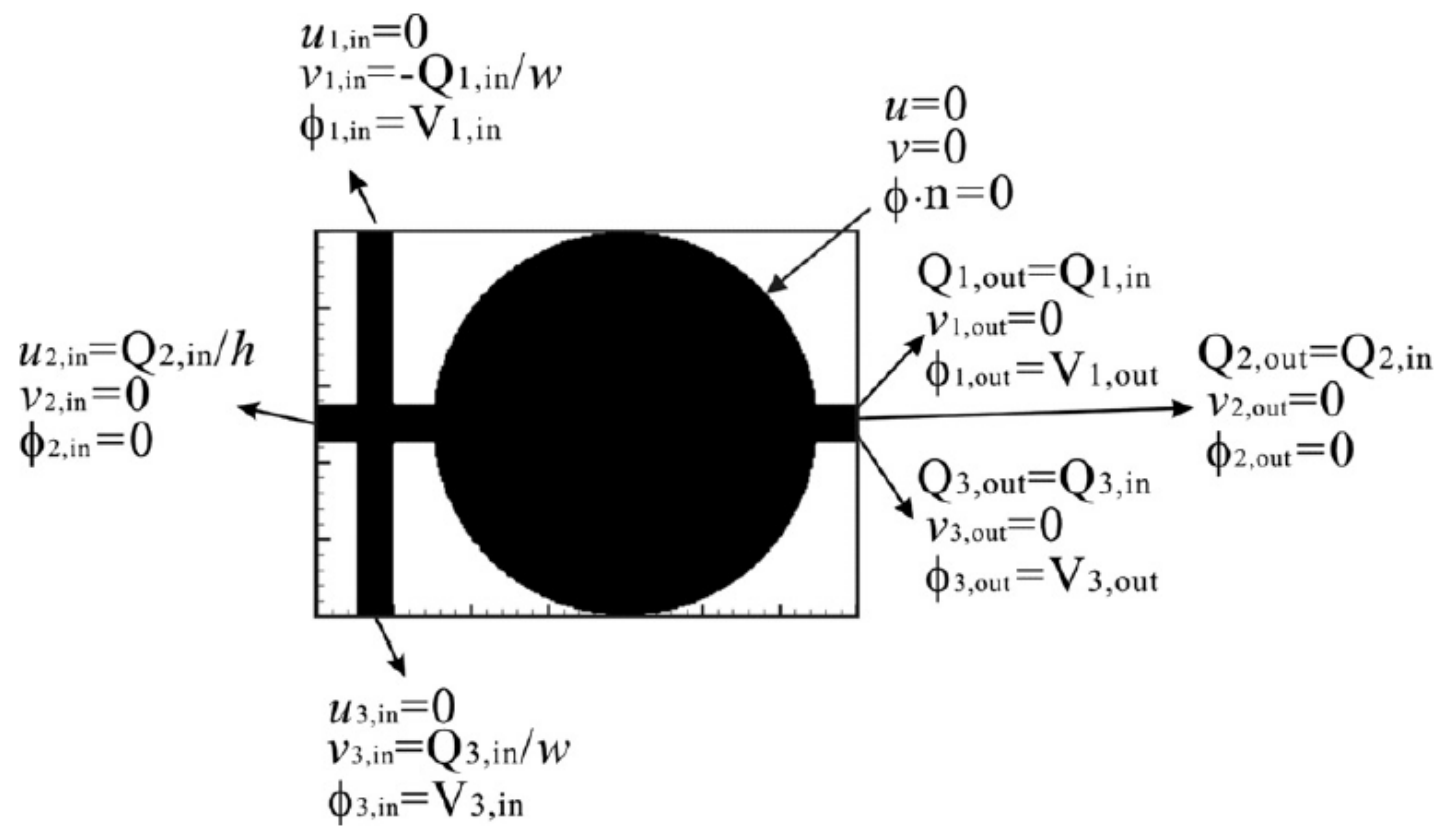

Figure 2 

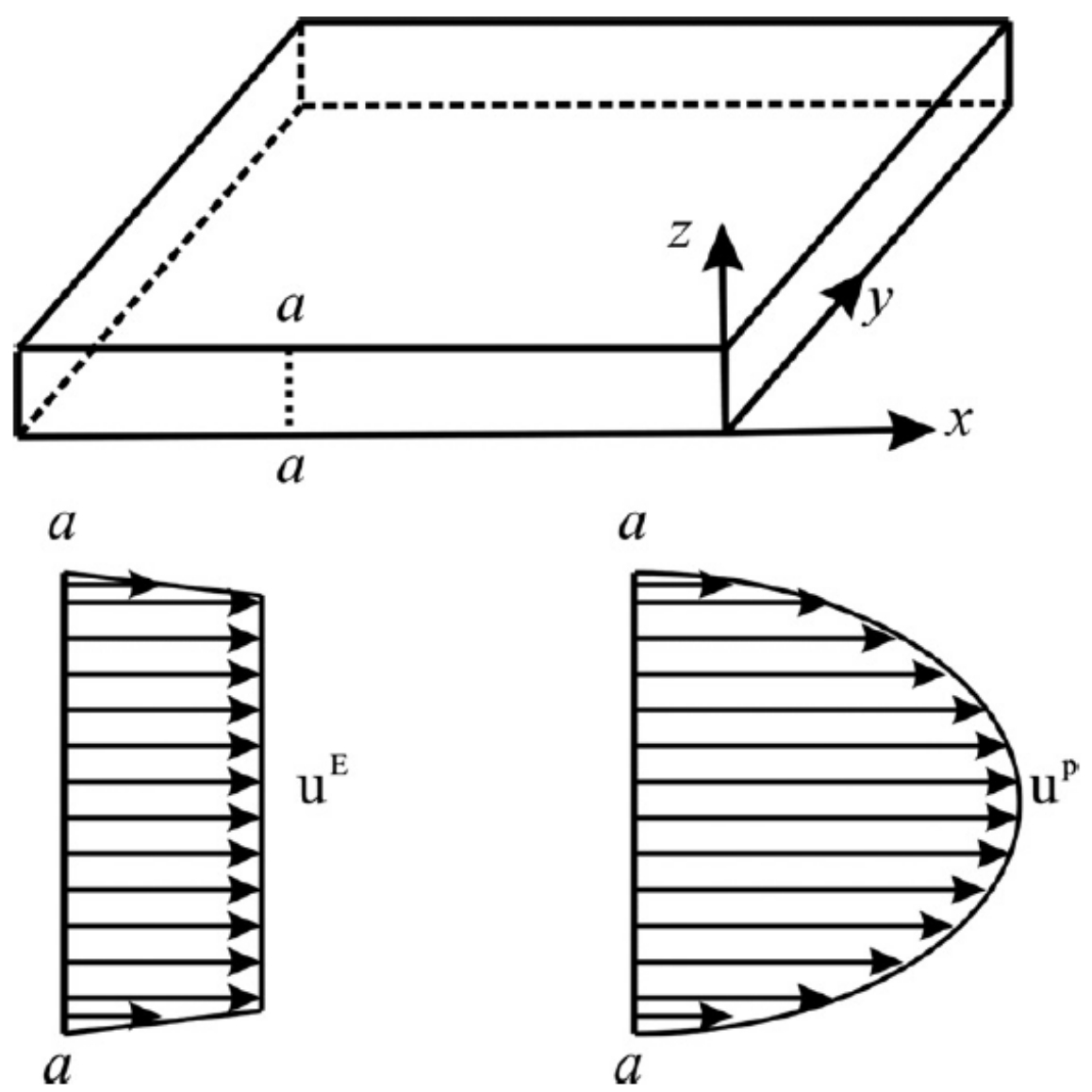

Figure 3 


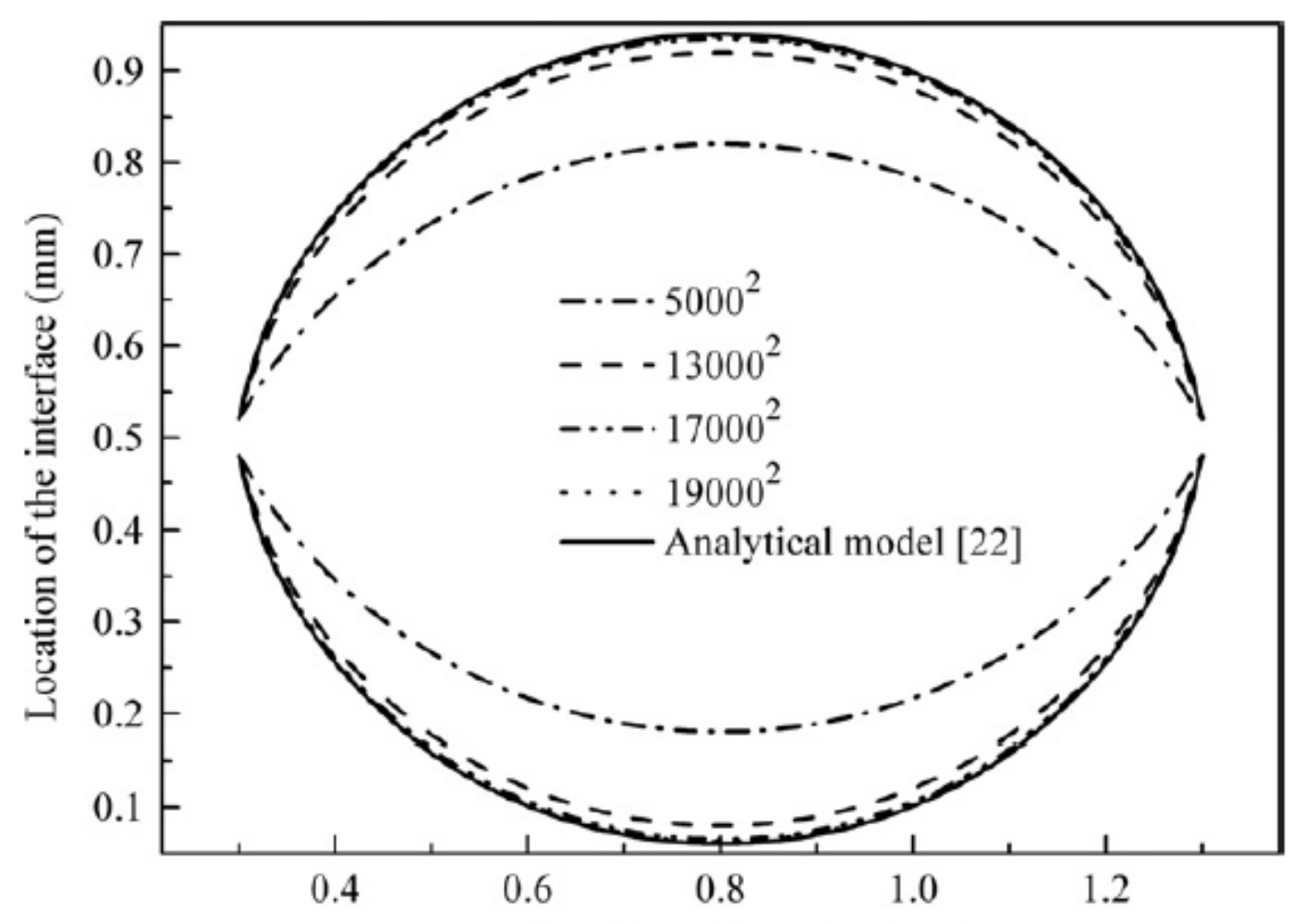

Figure 4 


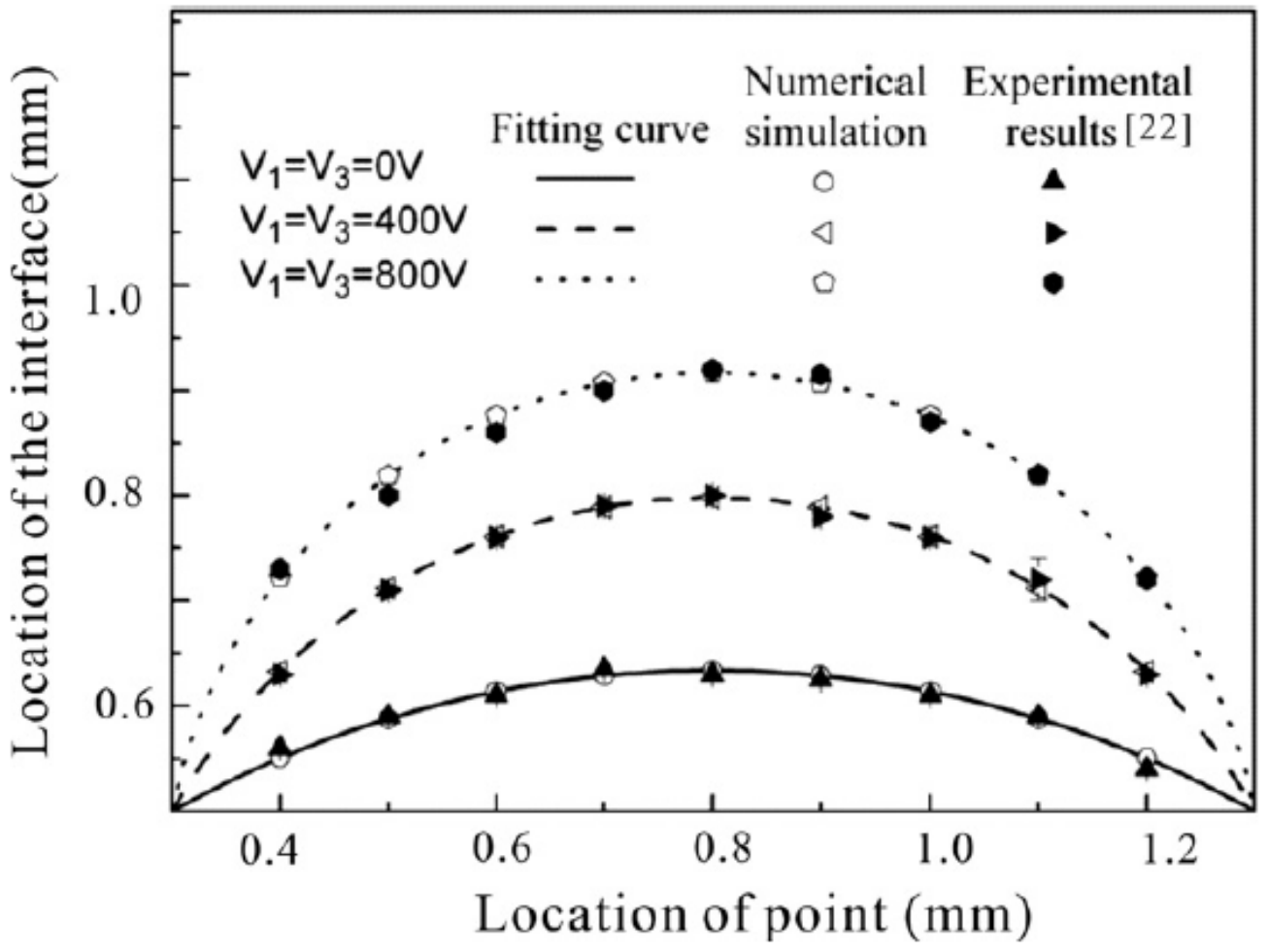

Figure 5 

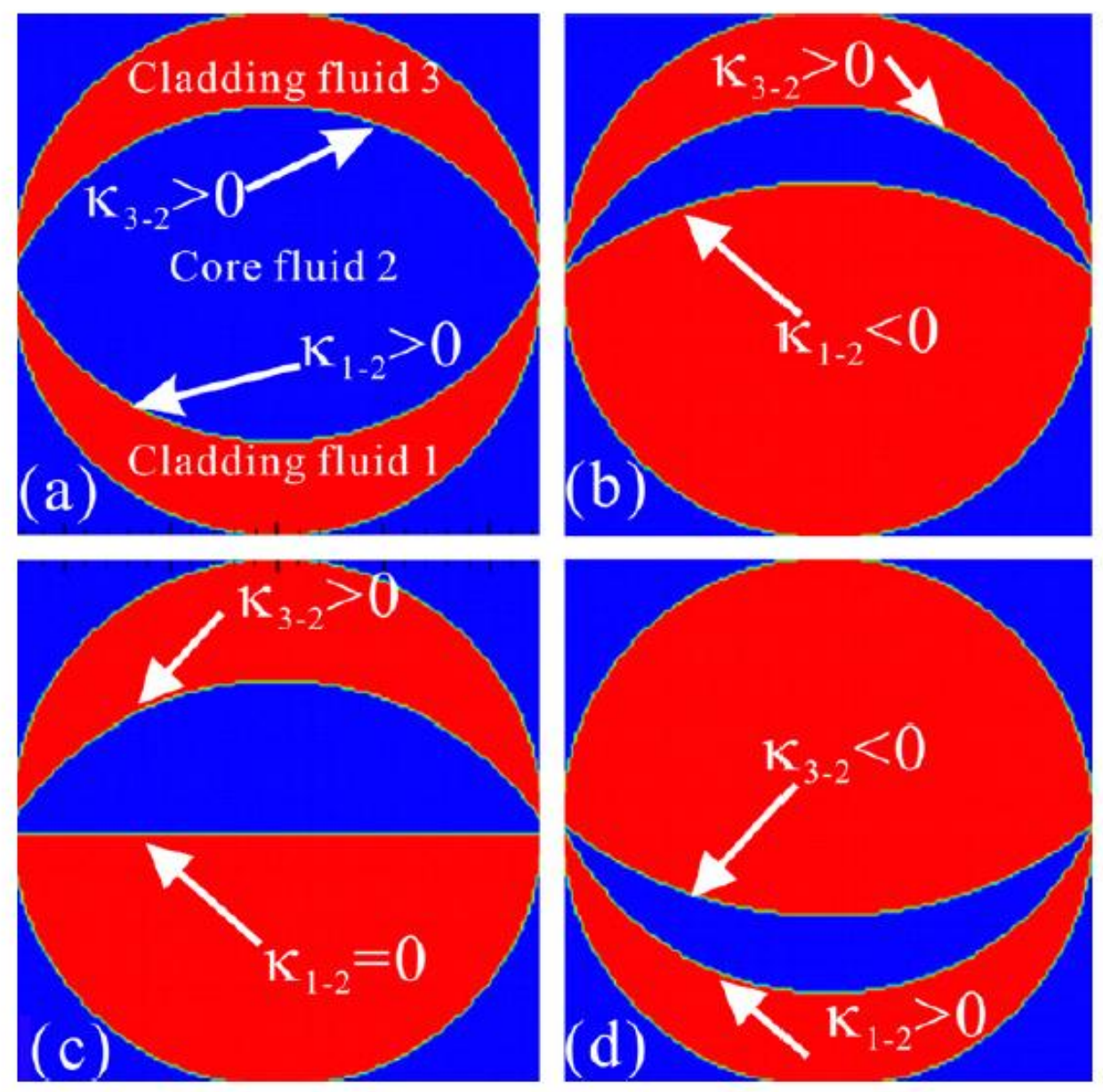

Figure 6 


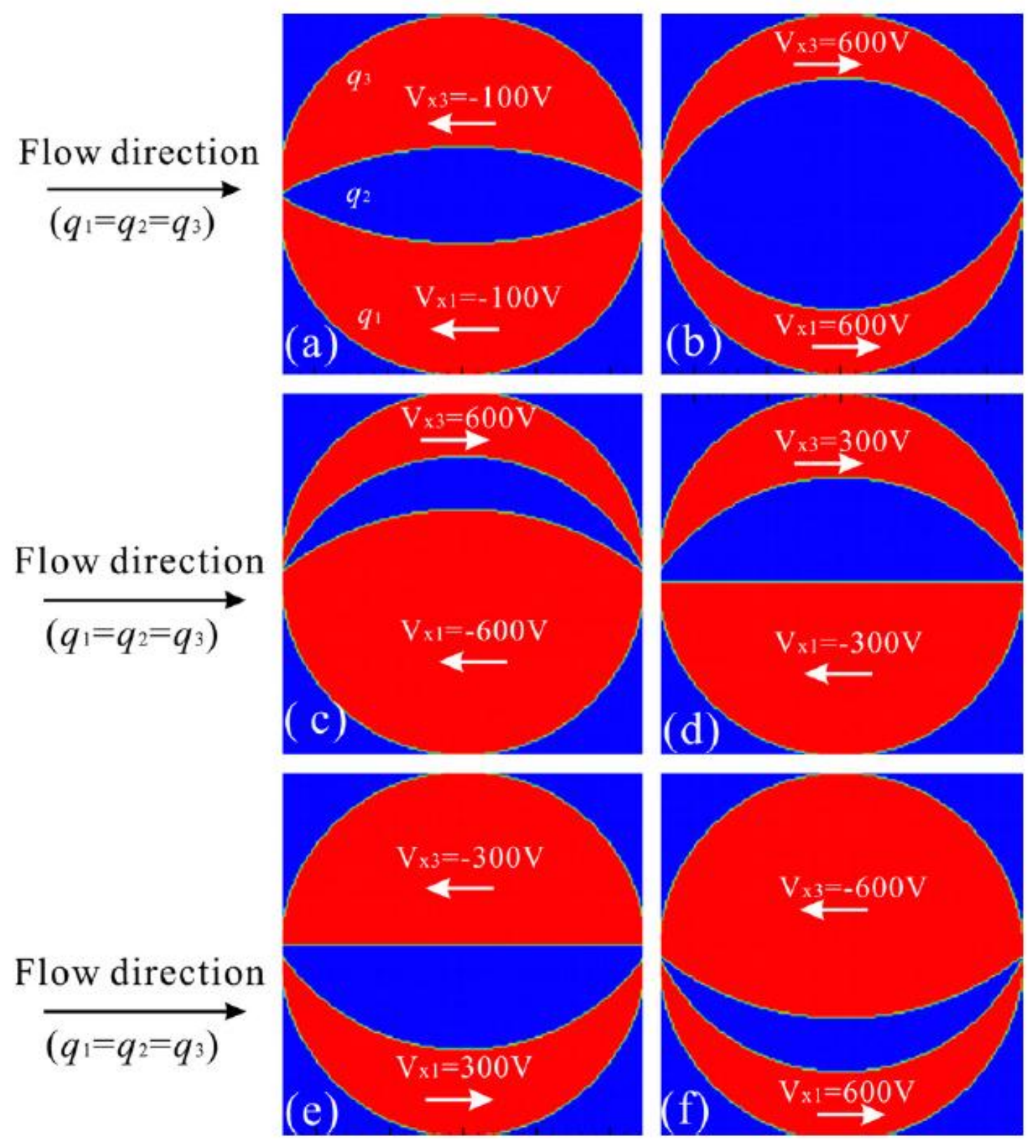

Figure7 


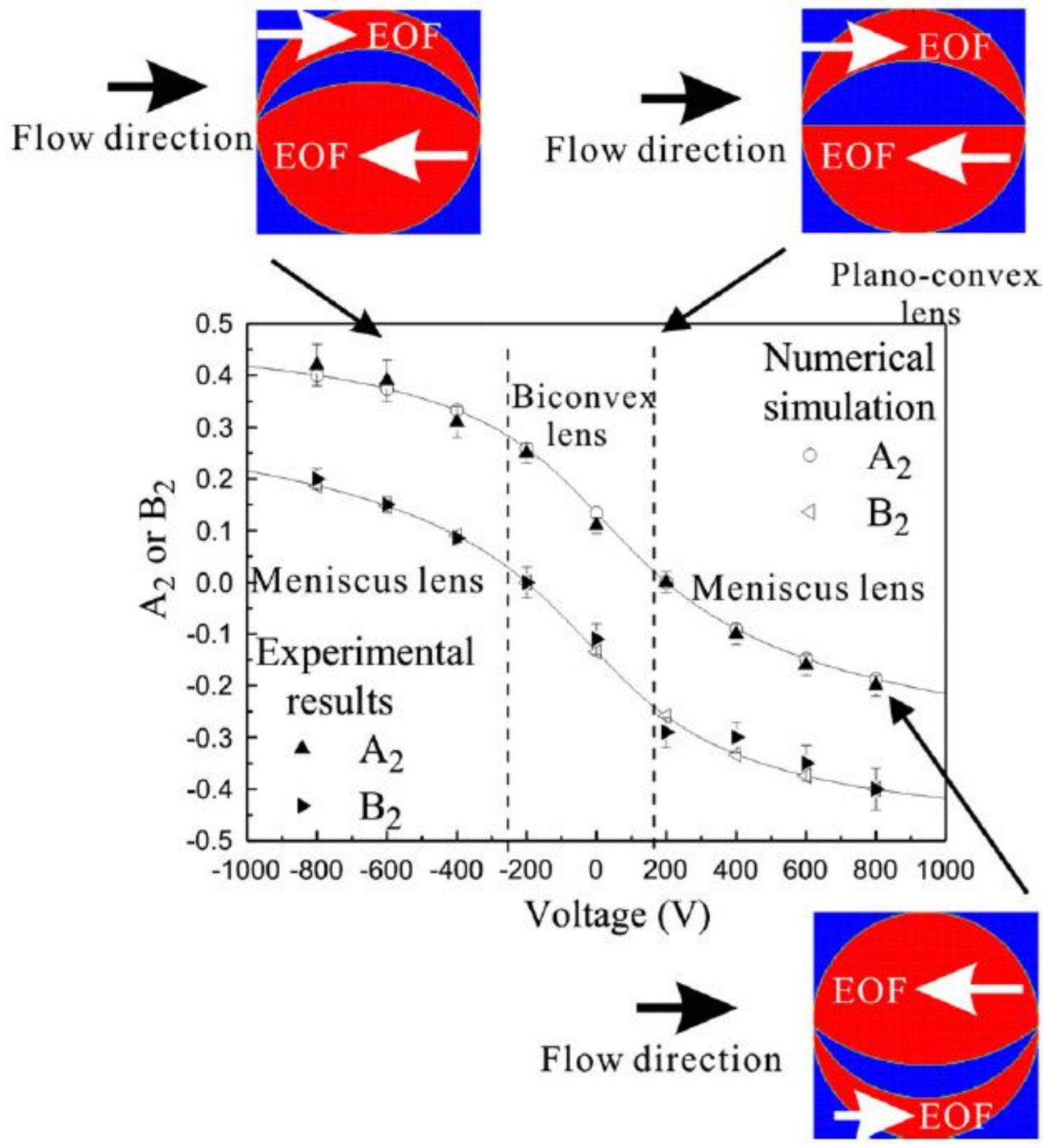

Figure 8 


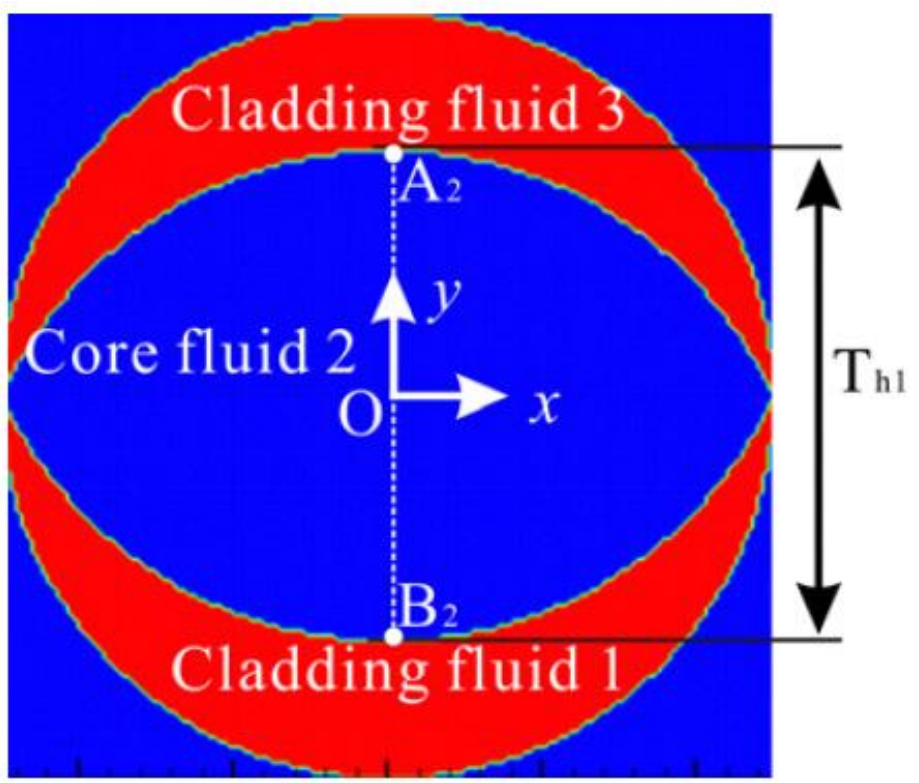

Figure 9 


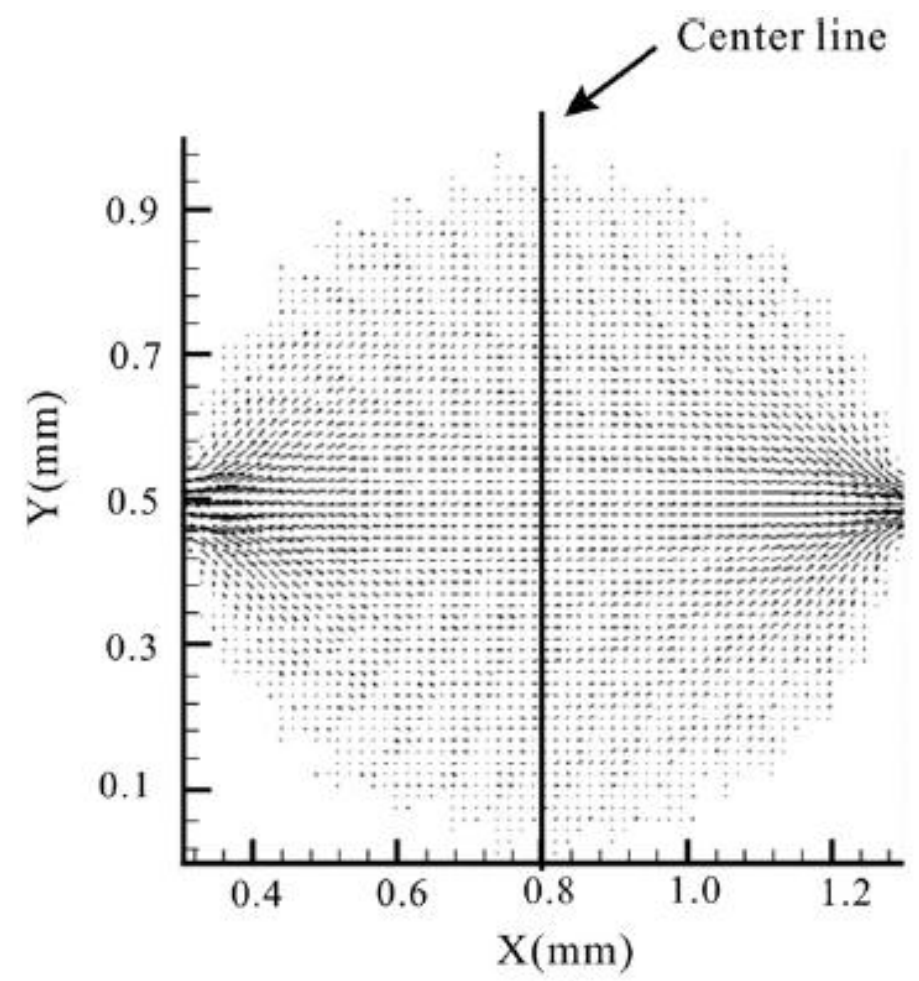

(a)

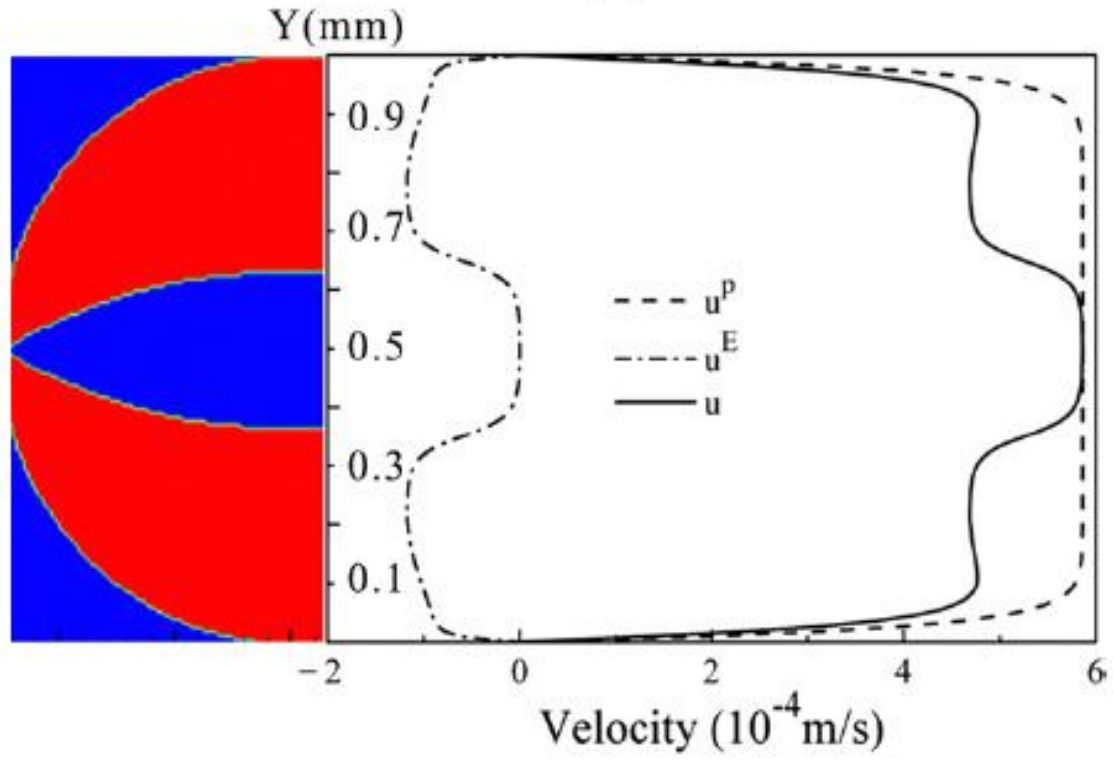

(b)

Figure 10 


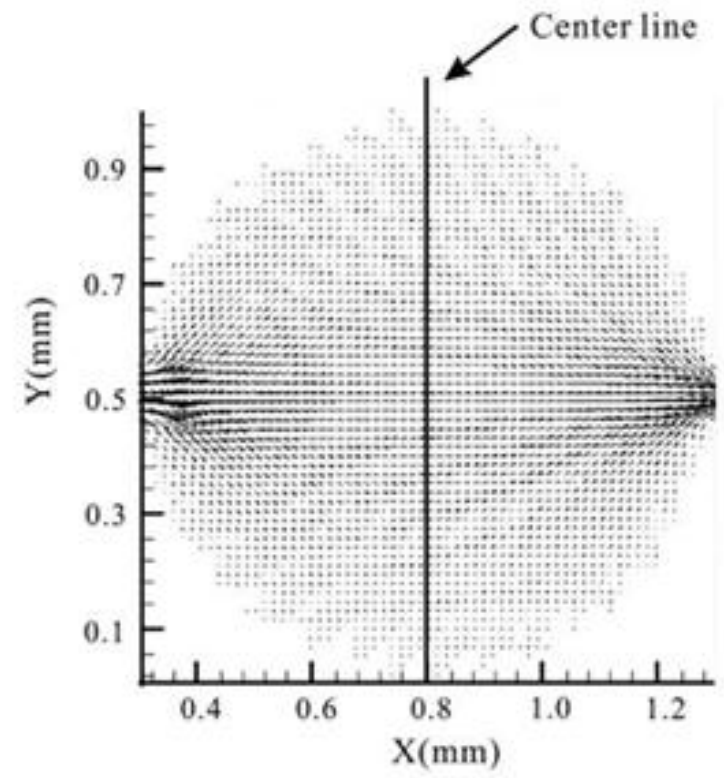

(a)

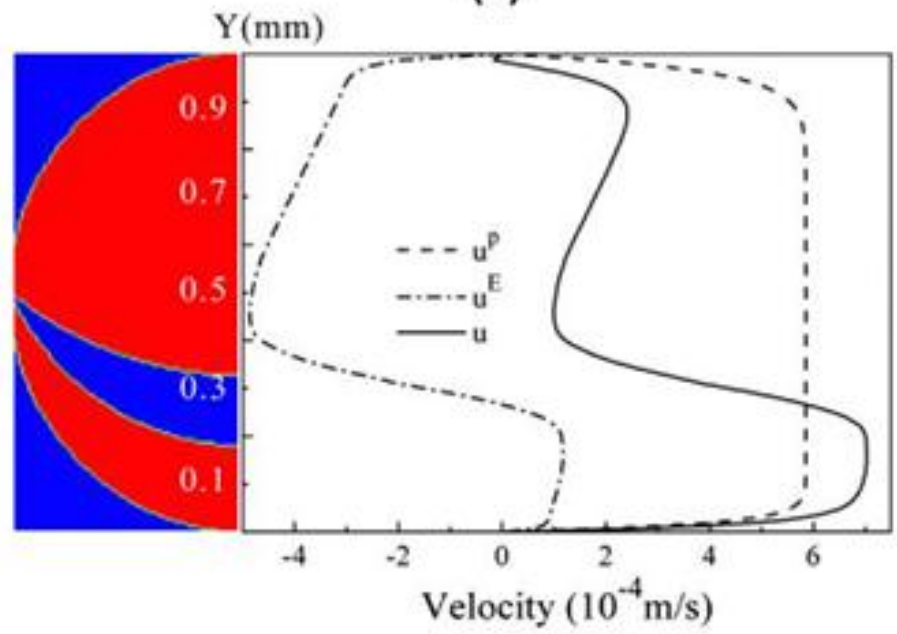

(b)

Figure 11 